International Journal of Engineering \& Technology, $7(2.25)(2018) 78-82$
International Journal of Engineering \& Technology
Website: $w w w . s c i e n c e p u b c o . c o m / i n d e x . p h p / I J E T$
Research paper

\title{
Design and implementation of blue tooth aided inexpensive safety band for fishermen
}

\author{
Jayarama Pradeep ${ }^{1}, S_{\text {S Sridharan }}^{2 *}$ \\ ${ }^{1}$ Professor, St.Joseph's College of Engineering, India \\ 2 Associate Professor, St.Joseph's College of Engineering, India \\ *Corresponding author E-mail: sridharsubbiah@gmail.com
}

\begin{abstract}
This paper presents with a new reliable inexpensive wireless health monitoring system designed for fishermen. Presently there are many smart devices available for the general population with many features in them that not put to use in every situation of our life. The most important aim of this paper is to deal with the safety and well-being of the fishermen during their voyage. The major cause of fishermen's poor health during voyage is due to lack of proper health guidance to the fishermen. This paper proposes with the monitoring of the fisherman's health through various sensors and relaying of those signals through blue tooth connectivity. The monitoring signals are finally obtained in Android mobile devices that can be used by the crew in real-time. The proposed approach is feasible for fishermen by establishing a software application to monitor the health of the fisherman. The proposed system is simple, economical and specifically designed for the people of the fishing community and it also enhances the motor control of the boat through the software interface by using a set of soft keys.
\end{abstract}

Keywords: Health Monitoring; Safety Band; Fishermen; Remote Patient Monitoring System (RPMS); Bluetooth.

\section{Introduction}

Fishing plays an important role in the economic development of our country and it has been a part of Indian culture since long. This industry has seen a constant growth in terms of production and profit; however the technological aspect still remains the same with limited applications. Association of Deep Sea Going Artisanal Fishermen (ADSGAF) developed concepts and products with engineering expertise. As a part of their projects, they aim to promote environmental friendly smart boats among the fishermen. These smart boats include a number of solar panels, sonar devices, bio-toilets, and many more technology which helps them during their journey as well as the environment. The mobile industry has taken over the world in its technological advancement in every other field around it.

Nowadays the use of mobile applications in various situations can be observed by us and with the advent of smart phones and smart bands there have been tremendous improvements in the technological development of the world. However these advancements have been seen in only the most economical and commercial sector of various industries like health, aviation, information and technology. Patient care and monitoring is poor in rural areas due to lack of technology which leads to patient death. To increase access to care and decrease healthcare delivery, a low cost monitoring system using android mobile devices like Remote Patient Monitoring System (RPMS) developed which provides the monitoring of patients in the home. This system monitored body temperature, pressure, heart beat, ECG. These parameters were compared with predetermined values. If any abnormal in these parameters, an alert is given to the relative of the patient. [1].

A smart system using wireless sensor network technology was designed to monitor the patient even from the remote location such as temperature, blood pressure and the pulse rate automatically. [2]. the impact of mental stress and needs to control were analyzed. A wearable, low power, and cost effective smart band was designed for health care. A machine learning algorithm was developed in which the various signals from different sensors are the inputs which has to be analyzed and correlated. [3]. For a wide range from remote healthcare monitoring to health management for all the residents in an aged facility, a new system was designed and monitored [4].

A health care system for various characteristics of the health condition was designed and implemented. It suffers some communication issues of a plug and play system[5]. A monitoring system was developed in which health condition such as temperature, blood pressure, sleep condition, weight, etc., over quite a long time had been monitored, saved and analyzed for four times per day [7]. A Wireless Wellness Monitor (WWM) which has a self monitored feedback system such as measuring devices, a home server as the base station, smart phone and databases which are connected through the internet has been developed by Bluetooth and Jini network [6-8]. Wireless Wearable Body Area Network (WWWBAN) was developed using GPRS and UTMS which is used to communicate the health signals. By means of Pervasive computing technologies, a Wellness Assistant system was designed using PC, cell phones and wrist watches for monitoring of health conditions [9-13]. An EEG devices was used by this researcher to identify the drowsiness level of an driver in order to prevent before any accident occur [14].

This paper deals with a new reliable health monitoring system for fishermen. The aim of this paper is to deal with the safety and wellbeing of the fishermen during their voyage. Currently there are many smart devices that are available for the general population with many features in them that are not put to use in every situation of our life and also they are not preferred by the fishermen due to their high costs and complexity of operations. The proposed paper 
aims to cut costs without compromising on the functionality, and also to provide a customizable feature filled smart device.

\section{Proposed system for safety band}

The proposed system is designed specifically for the fishermen community for their usage during their voyage into the system. It has minimal requirements of hardware as well as simple user software interface in the mobile application. It makes use of temperature sensor, pulse sensor and an accelerometer to measure the important vitals of the human body and also to monitor the position or state of action of the crew member. The data obtained from these sensors are relayed to the mobile software application through a Bluetooth module. The mobile software monitors the data relayed to it continuously and determines the status of the crew member. Figure 1 shows the design of system architecture of safety band using Atmega 328 Arduino controller.

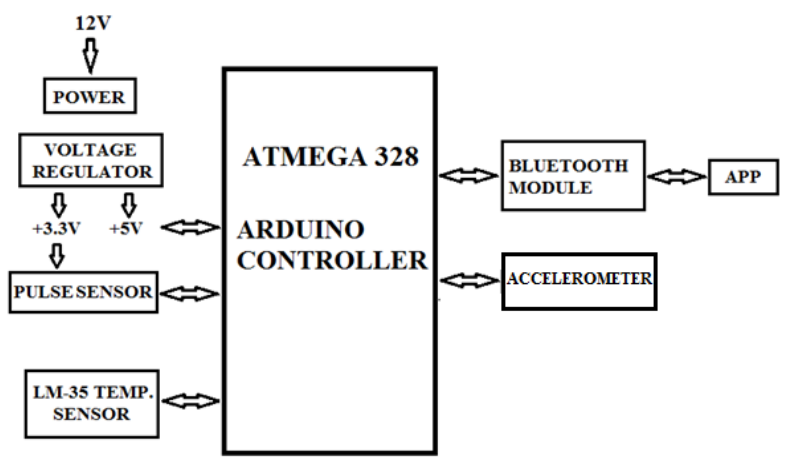

Fig. 1: System Architecture.

If the values obtained are not within the optimum values that are preset in it, then a warning can be issued in order to alert the crew captain. The proposed system is simple and efficient, user interface, specifically designed for the fishermen community for their voyage, Continuous monitoring system, no additional requirements such as high end smart phones, relatively low cost.

\section{Description of portable safety band}

The fisherman's health through various sensors are monitored by the safety band and relays through bluetooth connectivity. The monitored signal data can be used by the boat crew members in realtime to determine the health status of any crew member. This approach is feasible for fishermen by establishing a software application to monitor the health. This proposed system is simple, economical and specifically designed for the people of the fishing community and it also enhances the motor control of the boat, which is an additional feature of the band, through the software interface. To maintain the simplicity of the device, the safety band uses an Arduino developer board which is an open source coding system and for effective connectivity bluetooth module is used. In order to determine the health condition of any person, it is necessary to measure the vitals of that person; this includes the body temperature as well as the heart beat rate (BPM). This is achieved by using a temperature sensor LM 35 and a pulse sensor respectively.

In addition to these sensors an accelerometer is used as a part of the motor controller unit of the boat. The motor controller unit is an external unit that is placed in connection with the motor supply. The purpose of this unit is to power off the motor in case any member of the boat falls overboard during the voyage or while carrying out their works on the boat. It utilizes the values from the accelerometer which is programmed to detect a free fall condition. In case of any such event the micro-controller issues a command to the motor controller unit to stop the motor supply to prevent any further mishaps. The availability of the software interface in mobile and computing devices improves the user experience and the monitoring of the health of the crew member is easier. The software also includes a set of soft keys that are used in a manual control operation of the motor. The most advantageous aspect of our project is its compatibility and customizability of the functions and programs of the smart band. It can be easily enhanced as per the crew member's requirement. For the future enhancement, we aim to achieve a inter connectivity between fishermen boats and the coast guard patrol in order to improve the availability if medical assistances are required in case of any emergencies to personnel during voyage in deep sea. Also the accelerometer can be programmed in future to determine the actions performed by individual crew members, which could be useful in assigning tasks to the crew.

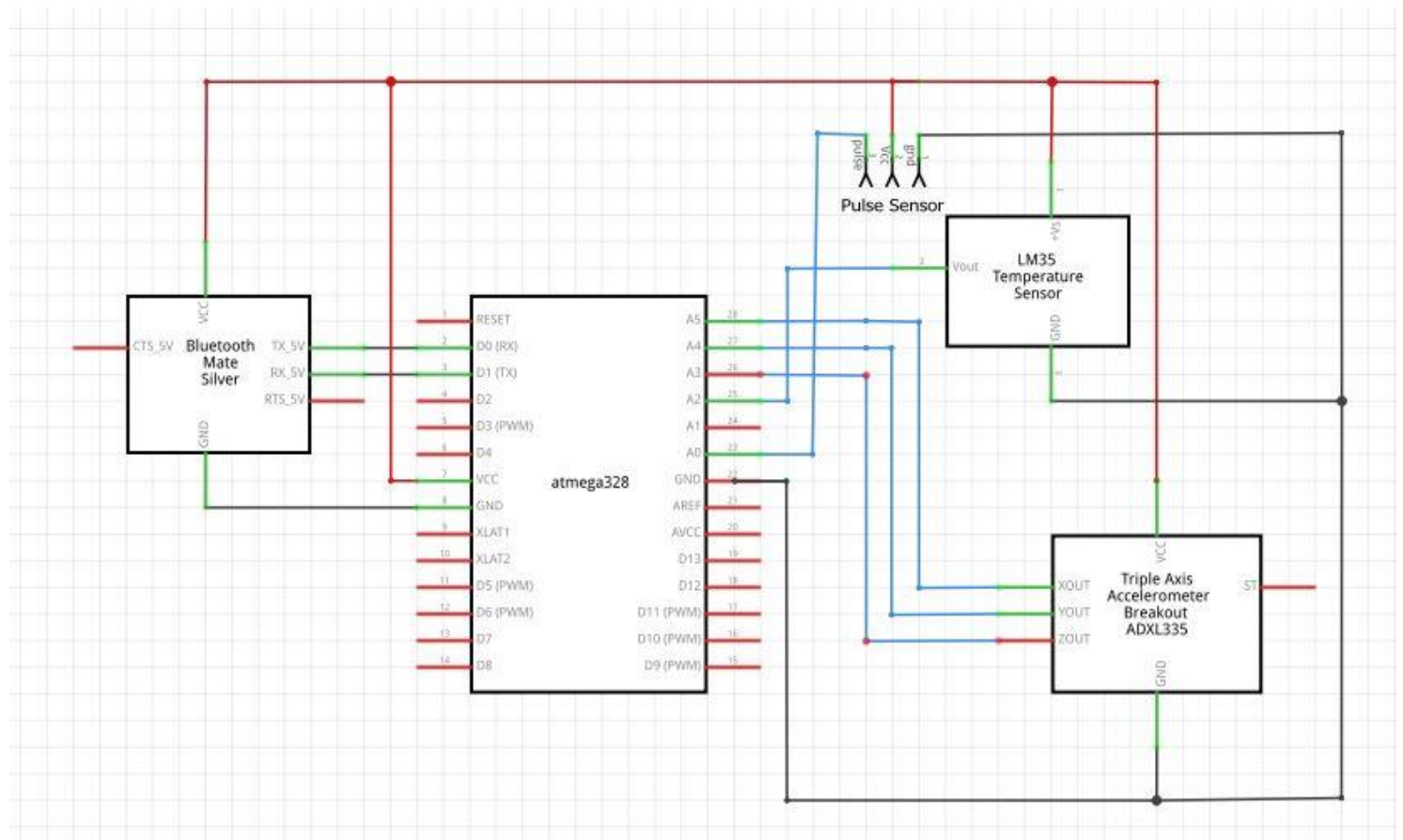

Fig. 2: System Circuit Diagram. 
Figure 2 shows the system circuit diagram which displays the connections of the various sensors and bluetooth to the micro controller. Here the VCC is the supply that is provided through the microcontroller to the sensors and bluetooth devices. GND is the grounding connection that is given to the devices. The Bluetooth transmission (TX) and receiver (RX) pins are connected to the micro controller's receiver (D0) and transmitter (D1) respectively. The pulse sensor output is given to the pin $\mathrm{A} 0$ of the microcontroller. The temperature sensor signal output (Vout) is connected to the analog pin of the micro controller (A2). The accelerometer signal values are given in terms of $\mathrm{X}, \mathrm{Y}, \mathrm{Z}$ axis respectively which are connected to the pins A5, A4 and A3 of the microcontroller. A microcontroller board based on the ATmega328P using Arduino / Genuino Uno is depicted in figure 3. This controller has 6 analog inputs, 6 PWM outputs and a $16 \mathrm{MHz}$ quartz crystal. It is connected to a computer with a USB cable or powers it with an AC-to-DC adapter or battery.

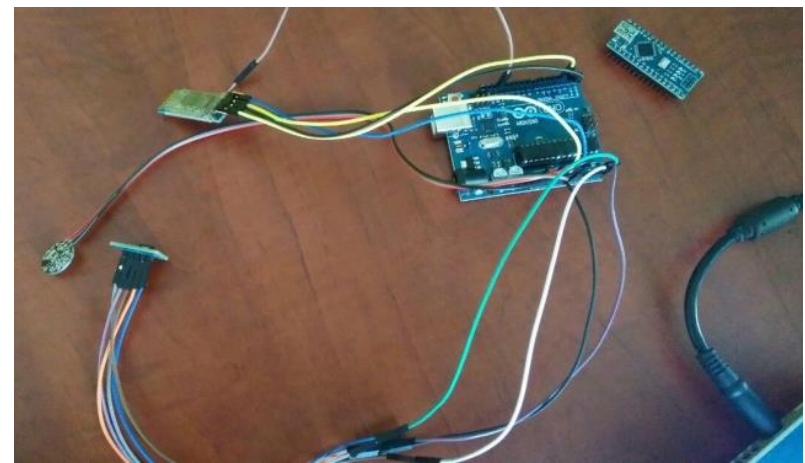

Fig. 3: Arduino Uno Developer Board.

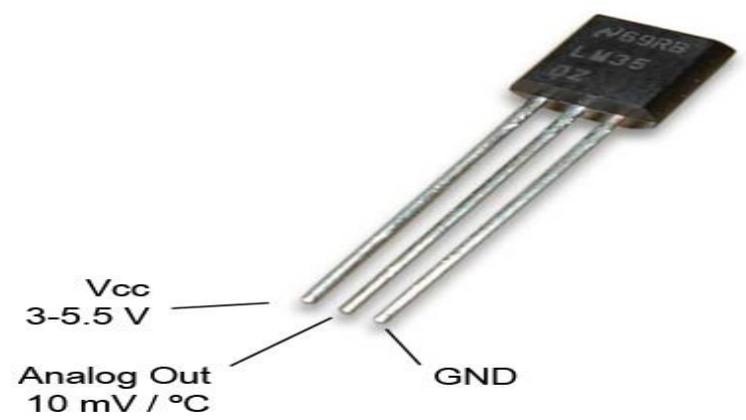

Fig. 4: Temperature Sensor.

A precision integrated-circuit temperature device using LM35 is as shown in the figure 4 . This sensor provides typical accuracy of $\pm 1 / 4^{\circ} \mathrm{C}$ at room temperature and $\pm 3 / 4^{\circ} \mathrm{C}$ over a full $-55^{\circ} \mathrm{C}$ to $150^{\circ} \mathrm{C}$ temperature range.

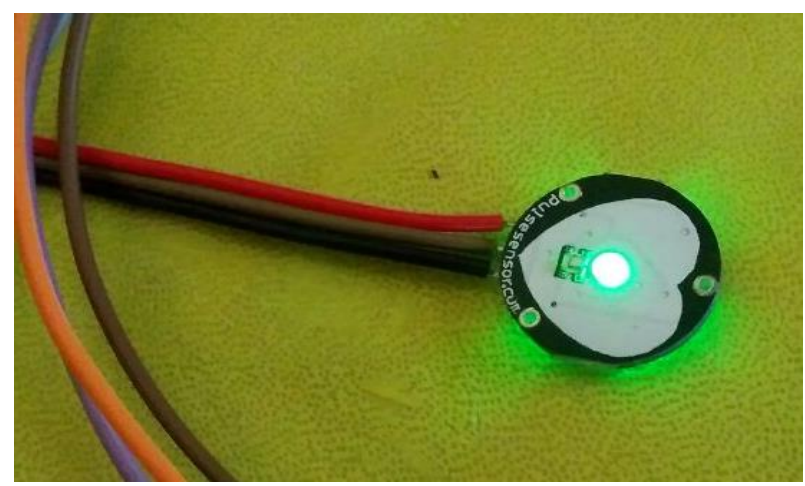

Fig. 5: Pulse Sensor.

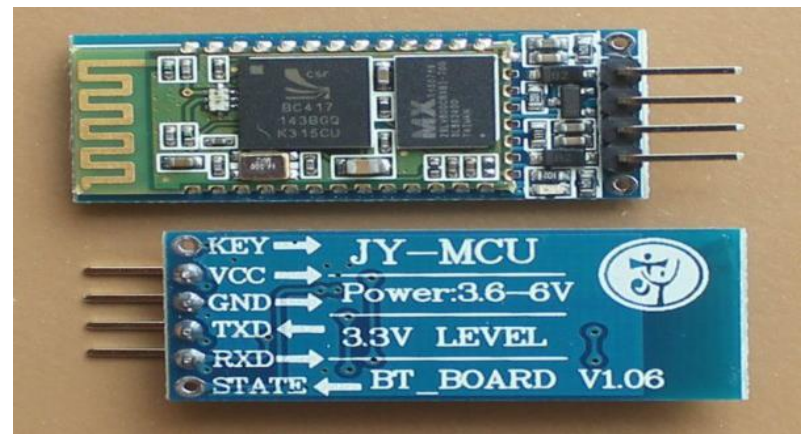

Fig. 6: Accelerometer Sensor ADXL335.

A plug-and-play heart-rate sensor (Pulse Sensor) for Arduino is as shown in the figure 5 and 6 . A optical heart rate sensor with amplification and noise cancellation circuitry are combined for making fast and easy operation. The ADXL335 in figure 6 is a small, thin, low power, complete 3 -axis accelerometer with signal conditioned voltage outputs.HC-05 module in Figure 7 is user friendly Bluetooth Serial Port Protocol (SPP) module which is used for transparent wireless serial connection setup.

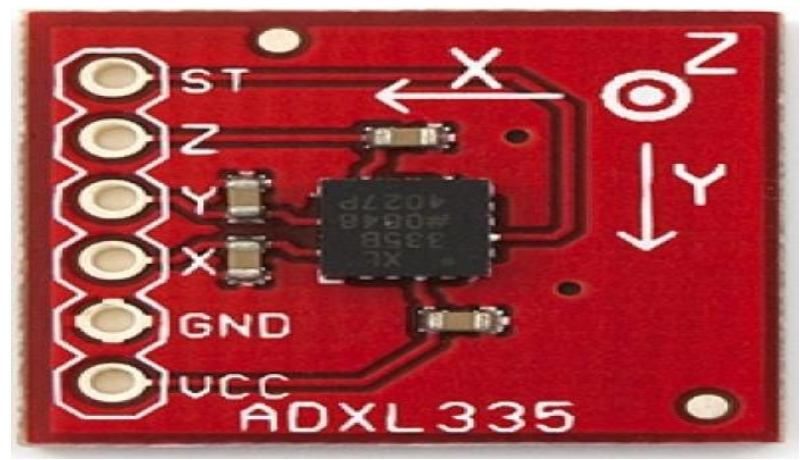

Fig. 7: HC 05 Bluetooth Module.

\section{System implementation}

\subsection{Implementation of Safety Band Unit}

The smart band is necessary to be worn on the boat by the fishermen at all times. The data is transmitted continuously to the mobile software that is available at the captain's cabin of the boat. This enables a continuous monitoring of the data from the crew members and to monitor their activities and health status. For the optimum comfortability and compact design, a miniature power source in the form of a battery is made utilized. In order to make available of the voltage conversions and power regulator LM7805 are used. For programming and testing purposes an Arduino Uno Starter kit and an AtMega 328 microcontroller were used. It can be observed that the sensors and Bluetooth modules are connected through connecting wires to the various assigned pins respectively as shown in figure8. The final components are soldered to an Arduino Nano which uses the same microcontroller and are designed to be placed around the wrist of the user as shown in figure 9 . 


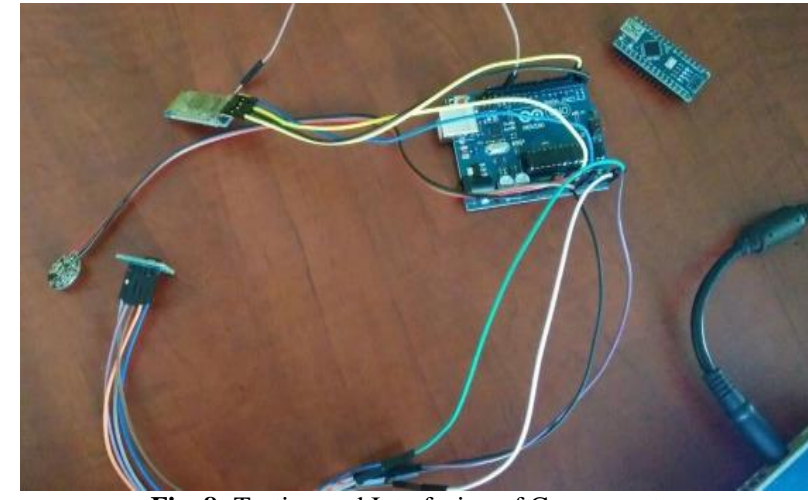

Fig. 8: Testing and Interfacing of Components.

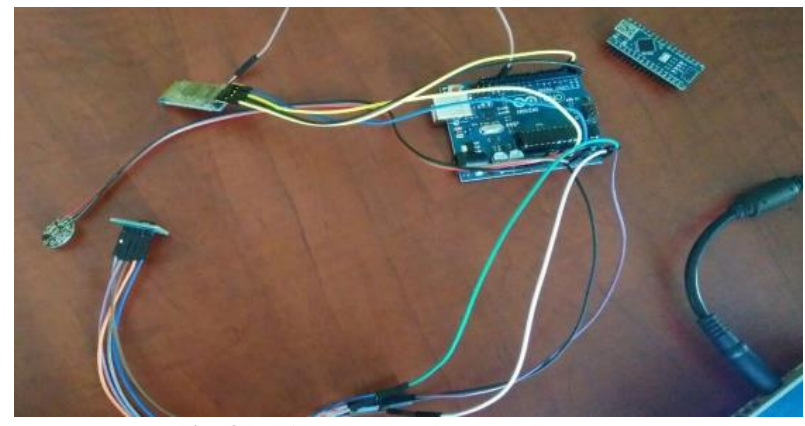

Fig. 9: Safety Band Using Arduino Nano.

\subsection{Implementation of motor control unit}

The motor control unit is an external unit that is placed in connection with the motor unit. This unit is interconnected to the software through another Atmega 328 microcontroller and a Bluetooth module that is present in this unit. The presence of this isolated unit of the safety band is to make use of an emergency stop of the fishing boat in order to avoid any mishap in case a crew member falls off board. This prevents the victim from being sucked into the propeller or from being hurt. A prototype of the motor controller unit is shown in Figure 11. In this prototype, a simple relay unit is used for the switching of the supply state from ON or OFF state, and a led is used for representation of the motor unit.

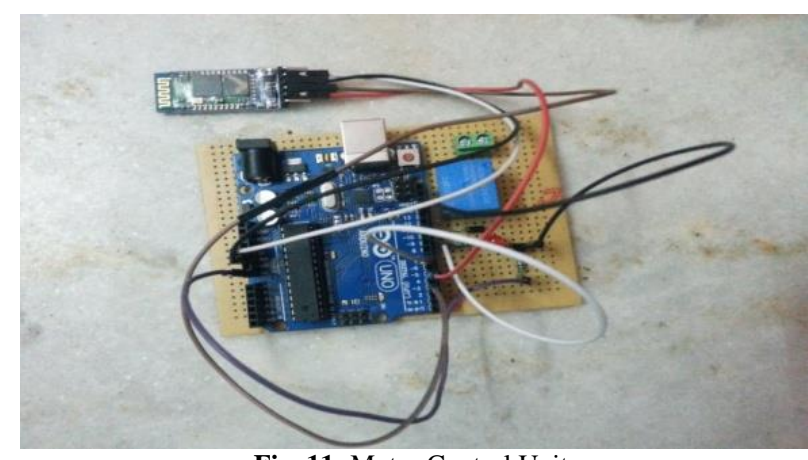

Fig. 11: Motor Control Unit.

Android Software Application

The software is maintained in the crew cabin in order to monitor the data continuously and compare it with the ideal values. Based upon the values obtained the software can be configured to suggest remedies that can be followed for the user in case of any health issue. The software interfacing of the motor control unit is maintained through the soft keys RUN SWITCH and KILL SWITCH, this allows the boat captain to have control over the motor unit for manual operations. If any crew member is observed to be falling overboard of the boat, then the software automatically sends the instruction to the motor control unit to turn the power supply off.

\section{Results and discussions}

Figure 13 shows the safety band output where pulse sensor LED glow and measures the heart rate, temperature, blood pressure per minute of the fishermen. The values of the temperature, heart beat are displayed in the monitor by using blue tooth and android software application which is shown in Figure 14. The values obtained in the band are stored and transferred to the mobile application software through the blue tooth module.

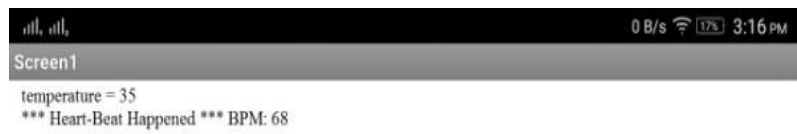

$$
\begin{aligned}
& \text { RUN SWITCH } \\
& \text { KILL SWITCH }
\end{aligned}
$$

Fig. 13: Safety Band Output.

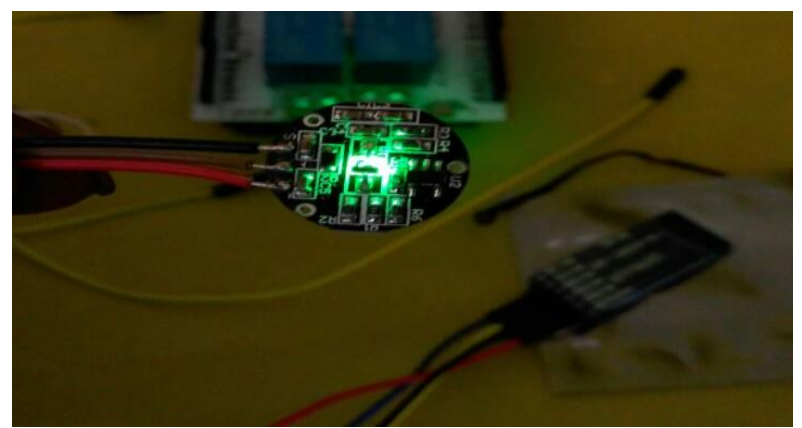

Fig. 14: Android Software Application Output.

The data are obtained in the software continuously and the monitoring takes place efficiently. In case of any severe accident or any fall overboard detection, the software takes the necessary steps to stop the boat immediately, thus avoiding any major injuries.

\section{Conclusion}

This paper proposes the design and implementation of bluetooth aided safety system for fishermen. This system is a simple, essential, cost effective to the fishermen community for safe travels during their voyage into the sea. It is a useful device for health monitoring and navigation, especially for fishermen. By modifying the design specifications, this device can be made miniature than proposed and efficiency can be improved by implementing more accurate GPS systems. This system can be used in preventing the small scale fishermen on crossing the International waters in future and can also be used as a distress alert system to the coast guard section whenever the fishermen face any calamities within the ocean. It is a social and commercial concerned system to help the fishermen and also to provide safety to them in the mid-sea.

\section{References}

[1] K. Mathan Kumar R.S. Venkatesan "A Design Approach to Smart Health Monitoring Using Android Mobile Devices”, IEEE International conference on Advanced Communication Control and Computing Technologies (ICACCCT), 8-10 May 2014.

[2] Kuruppuachchi, M.D.R. Perera and R.O.N. Meegama "Wireless Sensor Node for Simultaneous Monitoring of Health Parameters", IEEE International Conference on Advances in ICT for Emerging Regions (ICTER), 24-26 Aug. 2015, Sri Lanka. 
[3] SakineYalman, Muhammed Coşkun Irmak, Abdulsamet, "A Mobile-based approach to monitor biomedical signals" National Conference on Medical Technologies (TIPTEKNO), 15-18 October 2015, Turkey.

[4] Leroy L. Chan, Branko G. Celler, Nigel H. Lovell, "Development of a Smart Health Monitoring and Evaluation System" IEEE Region 10 Conference TENCON 2006, 14-17 November 2006, Hong Kong, China.

[5] D. Konstantas, A. van Halteren, R. Bults, K. Wac, I. Widya, N Dokovsky, G. Koprinkov, V. Jones, and R. Herzog. Mobile patient monitoring the mobihealth system. Stud Health Technol Inform, 103:307- 314, 2004.

[6] I. Korhonen, R. Lappalainen, T. Tuomisto, T. Koobi, V. Pentikainen, M. Tuomisto, and V. Turjanmaa. Terva: wellness-monitoring system. Engineering in Medicine and Biology Society, 20th Annual International Conference of the IEEE, 4(29):1988-1991, Oct 1998.

[7] A. Milenkovic, C. Otto, and E. Jovanov. Wireless sensor networks for personal health monitoring: Issues and an implementation. Computer Communications (Special issue: Wireless Sensor Networks: Performance, Reliability, Security, and Beyond), Elsevier, 29(13 14):2521-2533, Oct 2006.

[8] J. Parkka, M. van Gils, T. Tuomisto, R. Lappalainen, and I. Korhonen. Wireless wellness monitor for personal weight management. Information Technology Applications in Biomedicine, IEEE EMBS International Conference, pages 83-88, Nov 2000.

[9] N. Saranummi, I. Korhonen, M. van Gils, and S. Kivisaari. Barriers limiting the diffusion of ict for proactive and pervasive health care Proceedings of the IX MEDICON, Pula, Croatia, 4(29):1988-1991, Oct 2001.

[10] A. van Halteren, R. Bults, K. Wac, N. Dokovsky, G. Koprinkov, I Widya, D. Konstantas, V. Jones, and R. Herzog. Wireless body area networks for healthcare: the mobihealth project. Stud Health Techno Inform, 108:181-193, 2004.

[11] A. van Halteren, D. Konstantas, R. Bults, K. Wac, N. Dokovsky, G. Koprinkov, V. Jones, and I. Widya. Mobihealth: ambulant patient monitoring over next generation public wireless networks. Stud Health Technol Inform, 106:107-122, 2004

[12] U. Varshney. Pervasive healthcare and wireless health monitoring Jounral on Mobile Networks and Applications (Special Issue on Pervasive Healthcare), Springer, 12(2-3):111-228, June 2007.

[13] J. Yao, R. Schmitz, and S. Warren. A wearable point-of-care system for home use that incorporates plug-and-play and wireless standard. IEEE Trans Inf Technol Biomed, 9(3):363-371, Sep 2005.

[14] S. Pradeep Kumar and A. Wisemin Lins, "Brain Wave Sensor System for Accident Prevention in Vehicles", ARPN Journal of Engineering and Applied Sciences, Vol. 12, No. 8, April 2017, Pg No: 2455,2462 . 